This is the author's copy of the publication as archived with the DLR's electronic library at http://elib.dlr.de. Please consult the original publication for citation, see e.g.

https://link.springer.com/chapter/10.1007/978-3-030-38077-9 192

\title{
Design of a Multi-link Suspension for Motorcycles with a Sidecar
}

\begin{abstract}
Jakub Tobolar
For motorcycles, various suspension concepts for the front steerable wheel have been developed over decades. Even if the telescopic front fork has been eventually established as a common design, other alternative designs are still being evolved. A design of such an alternative - a multi-link suspension, particularly utilised for motorcycles with a sidecar, is discussed in the present paper. Beside some modelling aspects, optimisation of suspension's kinematics and benefits of an asymmetric layout for left and right cornering are elaborated.
\end{abstract}

\section{Copyright Notice}

(C2020 Springer Nature. Personal use of this material is permitted. Permission from Springer Nature must be obtained for all other uses, in any current or future media, including reprinting/republishing this material for advertising or promotional purposes creating new collective works, for resale or redistribution to servers or lists, or reuse of any copyrighted component of this work in other works.

Tobolar J. (2020) Design of a Multi-link Suspension for Motorcycles with a Sidecar. In:

Klomp M., Bruzelius F., Nielsen J., Hillemyr A. (eds) Advances in Dynamics of Vehicles on Roads and Tracks. IAVSD 2019. Lecture Notes in Mechanical Engineering. Springer, Cham. https://link.springer.com/chapter/10.1007/978-3-030-38077-9 192 


\title{
Design of a multi-link suspension for motorcycles with a sidecar
}

\author{
Jakub Tobolár \\ German Aerospace Center (DLR), Institute of System Dynamics and Control, \\ Wessling, Germany, \\ Jakub.Tobolar@DLR.de
}

\begin{abstract}
For motorcycles, various suspension concepts for the front steerable wheel have been developed over decades. Even if the telescopic front fork has been eventually established as a common design, other alternative designs are still being evolved. A design of such an alternative a multi-link suspension, particularly utilised for motorcycles with a sidecar, is discussed in the present paper. Beside some modelling aspects, optimisation of suspension's kinematics and benefits of an asymmetric layout for left and right cornering are elaborated.
\end{abstract}

Keywords: Motorcycle, multi-link suspension, modelling

\section{Introduction}

In the history of single track motor vehicles, various suspension concepts for the front steerable wheel have been developed. Even if the telescopic front fork has established itself as the preferable design, other solutions are possible and may appear advisable.

An invention from the early 1980s used two wishbones oriented in motorcycle's driving direction and connecting the front fork to the motorcycle's frame a system used by BMW and named duolever, see e.g. [1]. Yet another innovation is used under the name telelever by BMW as well. It connects the lower fork bodies right above the front wheel to the motorcycle's frame by means of one wishbone. The upper fork tubes are connected via a ball joint directly to the frame. A survey of alternative front suspension designs can be found in [2].

The benefits of such suspensions are especially the improvement of motorcycle's handling due to higher twisting stiffness of the front suspension, see [3], and/or the improvement of handling qualities during braking manoeuvres by reducing brake dive.

In the particular case of a motorcycle with a sidecar, the increased mass of the whole motorcycle combination and, hence, higher acting forces motivate additionally for alternatives. One of the preferable suspensions to fulfil the motorcycle combination requirements is a leading link fork [1].

The front forks discussed in the previous feature symmetric steering behaviour. Disadvantages of such property for motorcycles with sidecar and possible improvement utilizing an asymmetric multi-link suspension together with corresponding simulation results are presented in the following sections. 


\section{Multi-link front suspension}

The suspension designs described in the introduction have some parallels with independent suspensions of common passenger cars. For example, the abovementioned telelever compares to vehicle's McPherson suspension and the duolever is comparable to a double wishbone.

These affinities of the motorcycles' and automotive suspensions inspired the author to invent yet another automotive-like suspension - a multi-link - for front suspension of motorcycles with sidecar, see [4]. In the following, modelling aspects of such a multi-link suspension (hereinafter called multi-link fork) and optimisation of its kinematics for this particular scope of application are discussed.

\subsection{Multi-link fork design}

An actual automotive multi-link suspension - used especially for rear axles of luxury vehicles - consists of five links connecting the wheel upright with the vehicle's body [5]. The five links restrict the degrees of freedom to the upright's vertical compression and rebound only. If used for steerable automotive suspension, one of the links is considered a steering rod, thus enforcing the steering movement. For the motorcycle's multi-link fork, the four of five links are arranged in driving direction, see Fig. 1. The fifth link can induce the steering movement, as discussed below in Sec. 2.3.

Considering the motorcycle's longitudinal dynamics, the links are oriented pairwise in a way to optimize braking support angle $\epsilon_{B f}$, see Fig. 2. Together with the rear suspension's brake support angle $\epsilon_{B r}$ and the height $h_{C G}$ of the centre of gravity of the vehicle it is crucially responsible for the amount of the motorcycle's pitch movement during braking.

According to [1], the angle $\epsilon_{B}$ can be calculated by means of the translational velocity $\mathbf{v}_{W}^{*}$ at the "virtual" tire/road contact point $W$ as

$$
\tan \epsilon_{B}= \pm \frac{v_{W x}^{*}}{v_{W z}^{*}} .
$$

Such virtual contact point is considered on the wheel with blocking brake during wheel deflection and rebound. In the case of a conventional motorcycle with brakes mounted on the wheel carrier this means that the wheel can be virtually fixed on the fork during computer aided investigation of $\epsilon_{B}$. For practical reasons the point can simply be considered to be on the fork, too. This is also the case for the described multi-link fork.

The telescopic front fork is well known for its negative impact on the anti-dive effect. In contrast, both the duolever and the telelever mitigate such behaviour. Utilizing the multi-link fork, a motorcycle's front diving during braking can be tuned as well.

The lateral arrangement of the links can be done symmetrically to the longitudinal motorcycle's plane. Liu, see [6], elaborated such a design of the motorcycle's multi-link fork and demonstrated simulation results of various comparing 


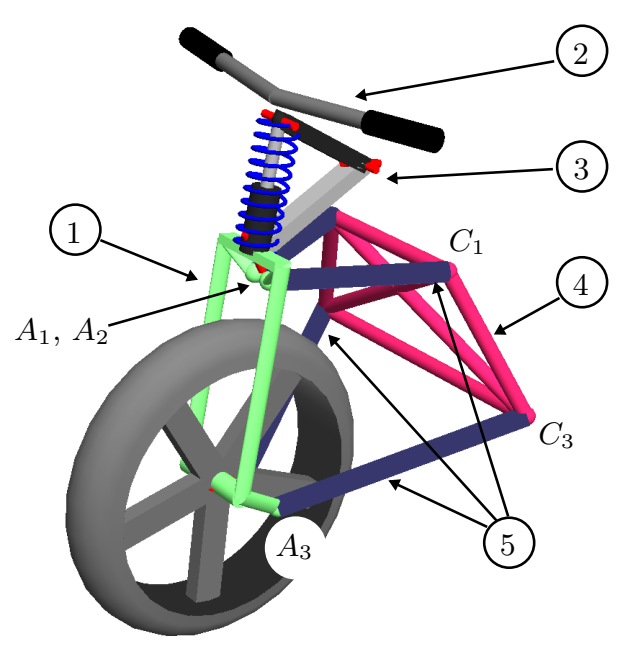

Fig. 1. Visualisation of the motorcycle's multi-link fork: fork (1), handlebar (2), steering assembly (3), motorcycle's frame (4) and linkage (5). The links' mounts on the motorcycle's body are marked $C_{i}$ with $i=1, \ldots, 4$ and those on the fork accordingly $A_{i}$ whereby the even numbers are on the left and odd numbers on the right side.

analyses. Utilizing an asymmetric design of the multi-link could be a doable way to deal with the particular steering handling of the (asymmetric) motorcycle with side car.

\subsection{Cornering behaviour}

Despite of descending from the single track motorcycle, the motorcycle with sidecar combination's handling is quite different. Due to its particular arrangement it even differ significantly from that of double track vehicles. This is especially due to the laterally asymmetric location of steered wheel and of driven wheel relative to the centre of mass of the whole motorcycle combination.

Considering the cornering at stability limits with the motorcycle having the sidecar on the right, the particular motorcycle combination arrangement typically yields a sidecar's wheel lifting ("flying the chair") in right turns. During left turning there is, on the contrary, a risk of the sidecar's nose diving. In both cases, a wheel contact loss accompanied with sudden handling change occurs which can lead to the lost of control over the vehicle.

The present work concentrates on performing at driving conditions far below such limits. Nonetheless, the asymmetric arrangement of the motorcycle combination still induces different behaviour during left and right cornering. This forces the driver to different handlebar actions for opposite turning. With the 


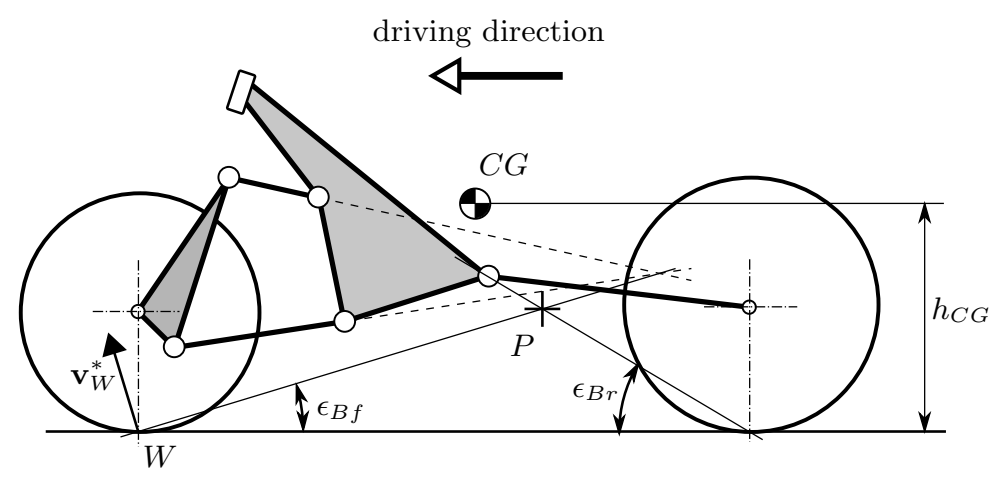

Fig. 2. On the calculation of front and rear braking support angles $\epsilon_{B f}$ and $\epsilon_{B r}$, respectively.

multi-link fork, both the steering assembly design and the asymmetric linkage could reduce such a behaviour.

\section{3 $\quad$ Steering assembly}

Various mechanical assemblies can be employed to transform the handlebar input to the front wheel. As mentioned in Sec 2.1, utilizing the fifth link would be an expected option which even enables to realise a variable steering angle transformation to diminish the differences between left and right turning. Nonetheless, this solution would also induce an unwanted variation of wheel's steering angle during suspension's compression and rebound even for constant handlebar input. This could be especially crucial for the motorcycle combination where payload - and consequently the suspensions' compression - can change significantly. Another drawback is the need for additional space to in any way connect the fifth link to the handlebar.

Therefore, a steering assembly with constant handlebar input transformation is preferred here. In [6], a drive shaft with two Hooke's joints and length change compensation is utilized for steering. Yet another mechanism are considerable. An option shown in Fig. 1 uses two rods connected by one laterally oriented revolute joint and a double Hooke's joint. Thus, the steering movement is restricted while the vertical movement is still available.

\subsection{Other elements}

Suspending elements can be arranged in various manners. In the variant shown in Fig. 1, there is one spring/damper unit placed above the wheel and connecting the motorcycle's fork with the motorcycle's frame beneath the handlebar. Even if other configurations are possible, they are not object of the study and will not be further elaborated here. 


\section{Simulation results}

To verify the theory discussed previously, a multi-body motorcycle model was established using Modelica [7] and analysed by means of simulations.

\subsection{Model of motorcycle with sidecar}

The investigated multi-body model comprises front and rear suspensions, wheels, frame with sidecar, rider and one sidecar's passenger. The steering assembly is being considered a part of the front suspension. The parameters of the motorcycle without sidecar are largely based on those given by [3]. In contrast to [3], neither frame elasticities nor the rider's upper body movement are considered. The parameters of the sidecar are estimated and summarised in Table 1. Stiffness and damping properties were adapted to fulfil vertical dynamics requirements for motorcycle combination with mass and its distribution different to [3].

Table 1. Sidecar's parameters.

\begin{tabular}{rr}
\hline Parameter & Value \\
\hline Mass & $85 \mathrm{~kg}$ \\
Passenger mass & $75 \mathrm{~kg}$ \\
Track & $1.1 \mathrm{~m}$ \\
Wheel longit. offset to rear wheel & $0.3 \mathrm{~m}$ \\
\hline
\end{tabular}

The tyre geometry considers tyre width and its circular cross-section according to [8]. While considering such a geometry is significant for proper modelling of motorcycle's behaviour at large tilting angles [9], it can generally be neglected for the motorcycles with sidecar as just minimum tilting angles occur. The multilink fork can, in contrast, induce significant wheel camber and, therefore, such tyre geometry is considered as well. Tire forces were modelled using the Pacejka's Magic Formula [10] to assess the vehicle dynamics behaviour.

In the analysed multi-link fork, the upper pair of links is located above the wheel whereby the lower pair is mounted under the wheel centre. Thus, a wide basis to support the braking torque is reached. Moreover, to place the upper linkage above the wheel allows for more space to optimize its lateral position.

\subsection{Cornering Assessment}

The vertical and longitudinal positions of the links' mounts were determined in a way to comply with braking requirements discussed in Sec. 2.1. The lateral positions of the mounts were found by means of an optimisation process in order to minimize the discrepancy between left and right turning. 
The simulated manoeuvre is quasi-stationary left and right cornering at constant velocity of $20 \mathrm{~km} / \mathrm{h}$ with monotonous increase of handlebar angle $\delta_{H}$. The discrepancy is given as difference between self-steering gradients $(S S G)$ of corresponding turns,

$$
S S G=\frac{\Delta \delta_{H}}{\Delta a_{y}},
$$

with $\Delta \delta_{H}$ being an increment of the handlebar angle and $\Delta a_{y}$ the corresponding increment of lateral acceleration.

The improvement of the self-steering behaviour of the motorcycle combination with optimized multi-link fork can be concluded from Fig. 3. In the upper diagram, the black lined $S S G$ of the motorcycle combination with multi-link fork reflects improvements over the full range of lateral acceleration compared to those of conventional motorcycle combination (indicated by red dashdotted lines). A particular improvement is achieved for $a_{y}$ between $\pm 2 \mathrm{~m} / \mathrm{s}$ where good symmetry of $S S G$ can be observed. This facilitates the driver riding straight on with small left and right handlebar corrections as such corrections evoke similar motorcycle's cornering reaction. A secondary effect is a slight increase of maximum lateral acceleration to be reached during right turn.

The lower diagram in Fig. 3 shows resulting $S S G$ for motorcycle combination cornering at higher constant velocity of $40 \mathrm{~km} / \mathrm{h}$. This was additionally investigated to prove the optimized multi-link fork at different conditions. The positive effects of the multi-link fork appear less distinctive but are still present.

\section{Conclusions}

A multi-link fork design was proposed to compensate for unfavorable effects during left and right cornering of motorcycles with sidecar. The suggested mechanical design complies with requirements on longitudinal and lateral dynamics. Particular attention was paid to achieve similar reactions during left and right cornering in order to relax driver's turning effort.

Presented simulation results confirmed improvements in cornering handling. Nonetheless, as the multi-link fork features far more tuning possibilities than considered in the present work, manoeuvres such as braking, braking in a turn or cornering at various velocities should be comprised in future optimisation.

\section{References}

1. Matschinsky W.: Radführungen der Straßenfahrzeuge. Springer-Verlag Berlin Heidelberg, 3rd edition, in German, doi: 10.1007/978-3-540-71197-1 (2007)

2. Mavroudakis B. and Eberhard P.: Analysis of alternative front suspension systems for motorcycles. Vehicle System Dynamics, 44:sup1 pp. 679-689, doi: 10.1080/00423110600883447 (2006)

3. Sharp R., Evangelou S. and Limebeer D.: Advances in the Modelling of Motorcycle Dynamics. Multibody System Dynamics, issue 3(12), pp. 251-283 (2004) 

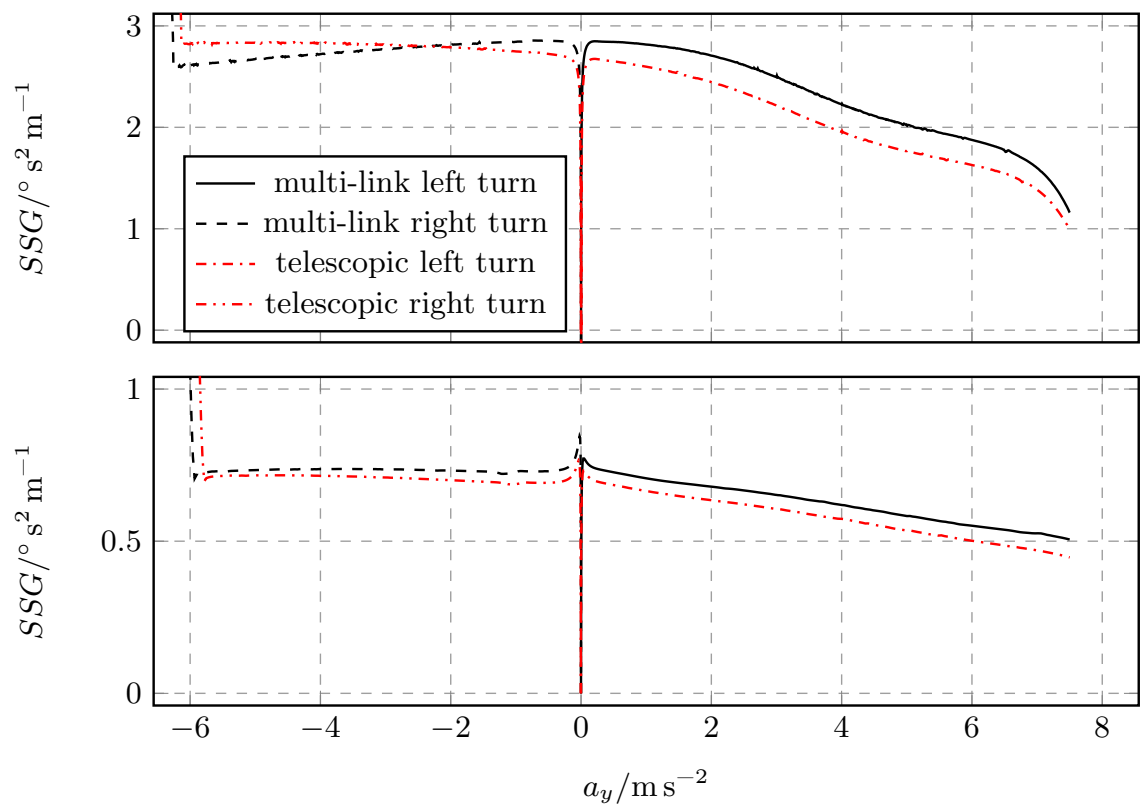

Fig. 3. Self-steering gradient $S S G$ over lateral acceleration $a_{y}$ for motorcycle with sidecar driving in a turn at $20 \mathrm{~km} / \mathrm{h}$ (above) and at $40 \mathrm{~km} / \mathrm{h}$ (below). Positive $a_{y}$ indicates left turn while negative values are for right turn.

4. Tobolar J.: Radaufhängung für ein lenkbares Rad. German patent DE 102012204031 B4 (2011)

5. Enke K.: Improvement of the ride/handling compromise by progress in the elastokinematic system of wheel suspension. In Road Vehicle Handling, I Mech E Conference Publications 1983-5, Torino (1983)

6. Liu Ch.: A multibody dynamics model of a motorcycle with a multilink front suspension. Electronic Theses and Dissertations. 7376. https://scholar.uwindsor.ca/etd/7376 (2017)

7. Otter M., Elmqvist H. and Mattsson S.E.: Multi-domain Modeling with Modelica. In CRC Handbook of Dynamic System Modeling, Chapman \& Hall, pp. 36.1-36.27, (2007)

8. Zimmer D. and Otter M.: Real-time models for wheels and tyres in an objectoriented modelling framework. Vehicle System Dynamics, 48:2, pp. 189-216, doi: 10.1080/00423110802687596 (2010)

9. Cossalter V.: Motorcycle Dynamics. 2nd edition, https://www.academia.edu/ 23761702/_Vittore_Cossalter_Motorcycle_Dynamics_BookZZ (2006)

10. Pacejka H. B.: Tire and Vehicle Dynamics. Elsevier Ltd, Oxford, 3rd edition, (2012) 